\title{
Visible and near-infrared spectral changes in plasma of psychiatric patients
}

\author{
YUKIKO HAKARIYA KATO ${ }^{1,2}$, HIDENORI MATSUNAGA ${ }^{3}$, \\ AKIKAZU SAKUDO $^{1}$ and KAZUYOSHI IKUTA ${ }^{1}$ \\ ${ }^{1}$ Department of Virology, Center for Infectious Disease Control, Research Institute for Microbial Diseases, \\ Osaka University, Yamadaoka, Suita, Osaka 565-0871; ${ }^{2}$ Clinical Center for Fatigue Science, 21 st Century COE Program, \\ Osaka City University Graduate School of Medicine, Abeno-ku, Osaka 545-8585; ${ }^{3}$ Department of \\ Psychiatry, Osaka General Medical Center, Osaka 558-8558, Japan
}

Received June 2, 2008; Accepted July 15, 2008

DOI: 10.3892/ijmm_00000050

\begin{abstract}
In the present study, the possible difference in visible and near-infrared (Vis-NIR) spectra of plasma in psychiatric patients compared to healthy control subjects was investigated. Mean Vis-NIR spectra of 60 psychiatric patients showed several specific peaks in the 630- to 930-nm region. Principal component analysis (PCA) and soft independent modeling of class analogy (SIMCA) were applied to manifest the spectral characteristics of each group. The PCA and SIMCA models demonstrated successful discrimination of the two groups using a score plot of the third versus fourth principal component and a Coomans plot, respectively. The PCA loadings were generally consistent with the discriminating power of the SIMCA model, indicating a specific pattern of Vis-NIR spectra in the plasma of psychiatric patients. The specific pattern possibly indicates altered superoxide, amine, and primary and secondary amides in plasma and may provide a possible indicator for the diagnosis of heterogeneous psychiatric disorders. This is the first step toward a clinical tool which has the potential to be applied in the diagnosis of psychiatric disease. Yet, there remain obstacles to its practical usage.
\end{abstract}

\section{Introduction}

Since Engel's theory of a bio-psycho-social model emerged in 1977 (1), descriptions of the clinical state of psychiatric disorders have accumulated. Currently, psychiatric diagnoses

Correspondence to: Dr Akikazu Sakudo, Department of Virology, Center for Infectious Disease Control, Research Institute for Microbial Diseases, Osaka University, 3-1 Yamadaoka, Suita, Osaka 565-0871, Japan

E-mail: sakudo@biken.osaka-u.ac.jp

Key words: visible and near-infrared, psychiatry, spectra have a descriptive nosology, that is, they are based on description rather than cause. This is because we do not yet know the mechanisms by which brain disorders lead to psychiatric illness. In this descriptive classification system, psychiatric disorders are defined by empirical data [Diagnostic and Statistical Manual of Mental Disorders, 4th edition, Text Revision (DSM-IV-TR) (2) and/or 10th revision of the International Classification of Diseases and Related Health Problems (ICD-10) (3)]. Therefore, this system has shaped current psychiatric research. However, recent reports have shown that there are many problems in clarity, logical coherence, factual content, redundancy, and ambiguity with DSM-IV-TR (4) and ICD-10 $(5,6)$. These problems have questioned the diagnostic validity and reliability of these methods and have severely influenced psychiatric research. Therefore, an objective diagnostic method for psychiatric disorders is warranted.

In this study, to test the hypothesis that the plasma of psychiatric patients has specific components, differences in visible and near-infrared (Vis-NIR) spectra of plasma between psychiatric patients and healthy donors were compared. A possible diagnostic method using Vis-NIR spectroscopy was explored by examining the differences in Vis-NIR spectra.

\section{Materials and methods}

Sample collection. Plasma samples from 60 psychiatric patients and 60 healthy donors were collected at the Osaka General Medical Center and the Medical Hospital of Osaka City University, respectively. All of the subjects were free of colds at the time of the collection. A venous-blood sample $(10 \mathrm{ml})$ from each subject was obtained from an antecubital vein. All samples were diluted 10 -fold with phosphatebuffered saline and adjusted to a constant volume $(1 \mathrm{ml})$ in a polystyrene cuvette (Sarstedt, Aktiengesellschaft, Germany) prior to Vis-NIR spectroscopy. This research project was approved by the Ethics Committee of Osaka General Medical Center and Osaka City University, and written informed consent was obtained from all psychiatric patients and healthy donors. 


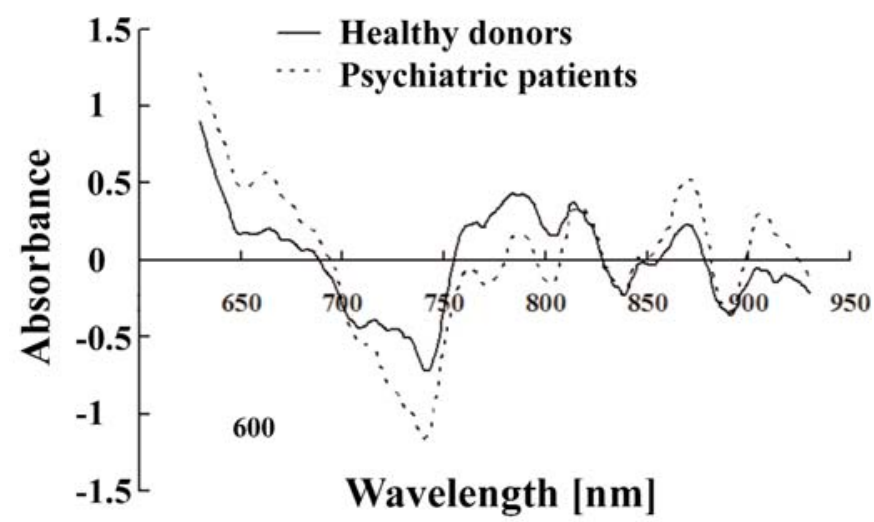

Figure 1. Visible and near-infrared (Vis-NIR) spectra of plasma from psychiatric patients and healthy donors. Transmittance Vis-NIR spectra of plasma from psychiatric patients and healthy donors were collected at wavelengths of 630-930 $\mathrm{nm}$ and subjected to smoothing and standard normal variates (SNV) to minimize noise and baseline shifts. The mean spectra of each group (healthy donors, solid line; psychiatric patients, dashed line) are shown.

Instruments, data collection, and data analysis. Vis-NIR spectra were measured at a resolution of $1 \mathrm{~nm}$ with an MCPD-7700 (Otsuka Electronics Co., Ltd., Japan). The temperature of the sample box was controlled with a water bath at $25^{\circ} \mathrm{C}$ during the Vis-NIR measurements. The spectral data were collected as absorbance values $[\log (1 / \mathrm{T})]$, where $\mathrm{T}$ is the transmittance in the range of wavelengths from 630 to $930 \mathrm{~nm}$. Pirouette software (ver. 3.11; Infometrix, Woodinville, WA) was employed for data processing. To minimize differences between spectra caused by baseline shifts and noise, preprocessing of Vis-NIR spectra, including mean-centering, smoothing (7) and/or standard normal variates (SNV) (8), was applied. The mean spectra of smoothing- and SNV-treated spectra were compared between psychiatric patients and healthy donors. To further identify the predominant absorbance peaks in the spectra, a principal component analysis (PCA) (9) and soft independent modeling of class analogy (SIMCA) (10) were also applied to develop models for the diagnosis of psychiatric disorders. For visualization of the PCA results, PCA score plots, which plot score values of two principal components (PCs), were used. The SIMCA results were visualized with a Coomans plot (11), which plots class distances against each other, to assess the classification performance of the SIMCA model by predicting class membership in terms of distance from the model. The critical distance from the model used corresponded to the 0.05 level and defined a 95\% tolerance interval. Through calculations of the PCA and SIMCA, loadings and discriminating power were obtained. The mathematical formulas of PCA, SIMCA, and preprocessing are available in the Pirouette manual. Statistical analyses of correlation and differences were performed by Spearman rank correlation analysis and Kruskal-Wallis test, where p-values $<0.05$ were considered significant.

Sample information. In PCA and SIMCA analysis, plasma samples from 45 psychiatric patients $(50.3 \pm 15.3$ years of age; male/female, 16/29), diagnosed on the basis of DSM IV-TR, and 45 healthy donors $(37.1 \pm 10.4$ years of age; male/female, $22 / 23$ ) were used as test samples to develop a calibration model, whereas samples from the other 15 psychiatric patients $(45.3 \pm 13.8$ years of age; male/female, $5 / 10)$ and 15 healthy donors $(36.3 \pm 12.2$ years of age; male/female, $5 / 10)$ were masked and used for predictions. Psychiatric patients were divided into 13 groups: schizophrenia (33.3\%), major depressive disorders (25\%), bipolar I disorders (20\%), generalized anxiety disorders $(3.3 \%)$, undifferentiated somatoform disorders $(3.3 \%)$, dysthymic disorders (3.3\%), pain disorder $(1.7 \%)$, hypochondriasis $(1.7 \%)$, somatization disorder $(1.7 \%)$, hypochondriasis with pain disorder $(1.7 \%)$, posttraumatic stress disorder $(1.7 \%)$, bipolar I disorder with psychotic features (1.7\%) and limbic encephalitis (1.7\%).

\section{Results}

A total of 120 subjects were examined: 60 subjects with psychiatric disorders $(49.1 \pm 15.0$ years of age; male/female, $21 / 39)$ (Table I) and 60 healthy donors $(36.9 \pm 10.8$ years of age; male/female, 27/33). Among the subjects with psychiatric disorders, one patient requested counseling from a clinical psychologist but was not medicated at the time of blood collection. The medications of the other subjects with psychiatric disorders were : major and minor tranquilizers $(n=29)$, minor tranquilizer only $(n=5)$, minor tranquilizer + antidepressant $(n=5)$, major tranquilizer only $(n=3)$, major and minor tranquilizers + antidepressant $(n=3)$, minor tranquilizer + lithium carbonate $(n=3)$, major tranquilizer + antidepressant $(n=1)$, minor tranquilizer + antidepressant + lithium carbonate $(n=1)$, major and minor tranquilizers + antidepressant + lithium carbonate $(n=1)$, major and minor tranquilizers + lithium carbonate $(n=1)$, minor tranquilizer + hormonal agent + vitamins + others $(n=1)$, major and minor tranquiliter + antiphlogistic analgetic $(n=1)$, major and minor tranquilizers + antidepressant + antiphlogistic analgetic + others $(n=1)$, major and minor tranquilizers + antiphlogistic analgetic + muscle relaxtant + others $(n=1)$, major and minor tranquilizers + antiphologistic analgetic + anticoagulant + others $(n=1)$, major and minor tranquilizers + antihypertensive + others $(n=1)$, and hormonal agent $(n=1)$.

As raw Vis-NIR spectra showed noise and a baseline shift, it was difficult to find any significant difference between psychiatric patients and healthy donors. Thus, to minimize noise and baseline shift, Vis-NIR spectra of plasma from psychiatric patients and healthy donors were subjected to smoothing and SNV. Then, mean spectra of the smoothed and SNV-treated spectra were compared between the two groups. Several peaks were found in both groups in the region 630-930 nm, while slight differences were observed between the groups in this region. In particular, there were spectral differences between the groups in the regions 650-675, 720740, 770-790, 850-875 and 900-925 nm (Fig. 1).

Next, spectra were further subjected to PCA and SIMCA. Clear discrimination of the plasma samples of psychiatric patients from those of healthy donors was noted in PCA scores using the third principal component (PC3) and fourth principal component (PC4) (Fig. 2A). The SIMCA model using test samples also allowed correct separation of VisNIR spectra of 43 of $45(95.6 \%)$ healthy donor plasma and 
A

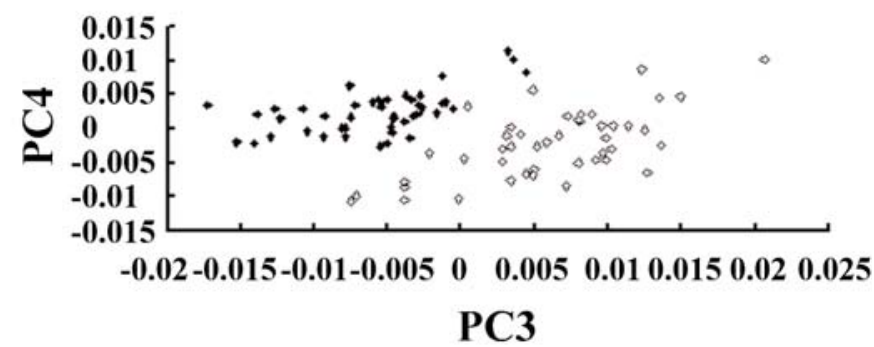

B

\section{Masked samples}

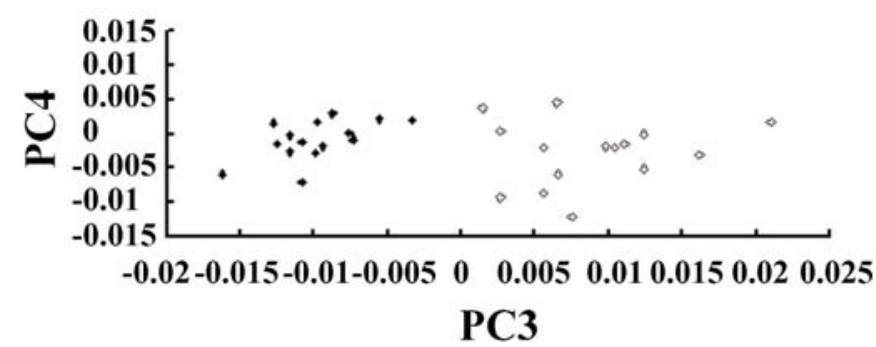

C

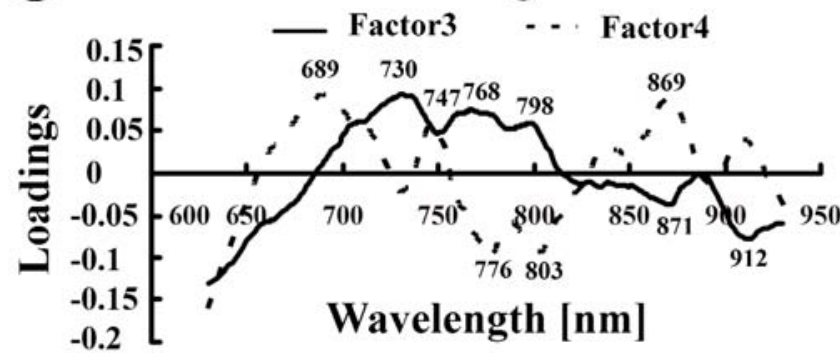

Figure 2. Principal component analysis (PCA) of the Vis-NIR calibration model for test samples and the predictions for masked samples using plasma of psychiatric and healthy donors. Plasma samples from healthy donors and psychiatric patients were subjected to Vis-NIR spectroscopy. After the collection of Vis-NIR spectra of plasma samples, the spectral data were preprocessed by mean centering and smoothing and subjected to PCA calibration modeling to develop a multivariate model to distinguish between healthy donors and psychiatric patients. The PCA score plot of the third principal component (PC3) versus the fourth principal component (PC4) for Vis-NIR spectra of test samples (A) and masked samples (B) from healthy donors and psychiatric patients by the PCA model showed a clear distinction between healthy donors (closed diamonds) and psychiatric patients (open diamonds). (C) PC3 (solid line) and PC4 (dashed line) loadings of the PCA.

45 of $45(100 \%)$ psychiatric patient plasma samples (Fig. 3A). SIMCA using the Coomans plot demonstrated that plasma classes from healthy donors and psychiatric patients did not share multivariate space, providing validation for the separation (Fig. 3A). Furthermore, the masked samples were subjected to Vis-NIR spectroscopy and further predictions with the PCA and SIMCA models. Complete discrimination of the masked plasma samples between psychiatric patients and healthy donors was attained using the PCA and SIMCA models of Vis-NIR spectra (Figs. 2B and 3B). PCA showed a clear distinction of the masked samples between healthy donors and psychiatric patients. SIMCA predicted 14 of 15 $(93.3 \%)$ healthy donors and 14 of $15(93.3 \%)$ psychiatric patients based on Vis-NIR spectra from masked plasma samples. In this model, the effect of medication could be ruled out, because subjects with psychiatric disorders included
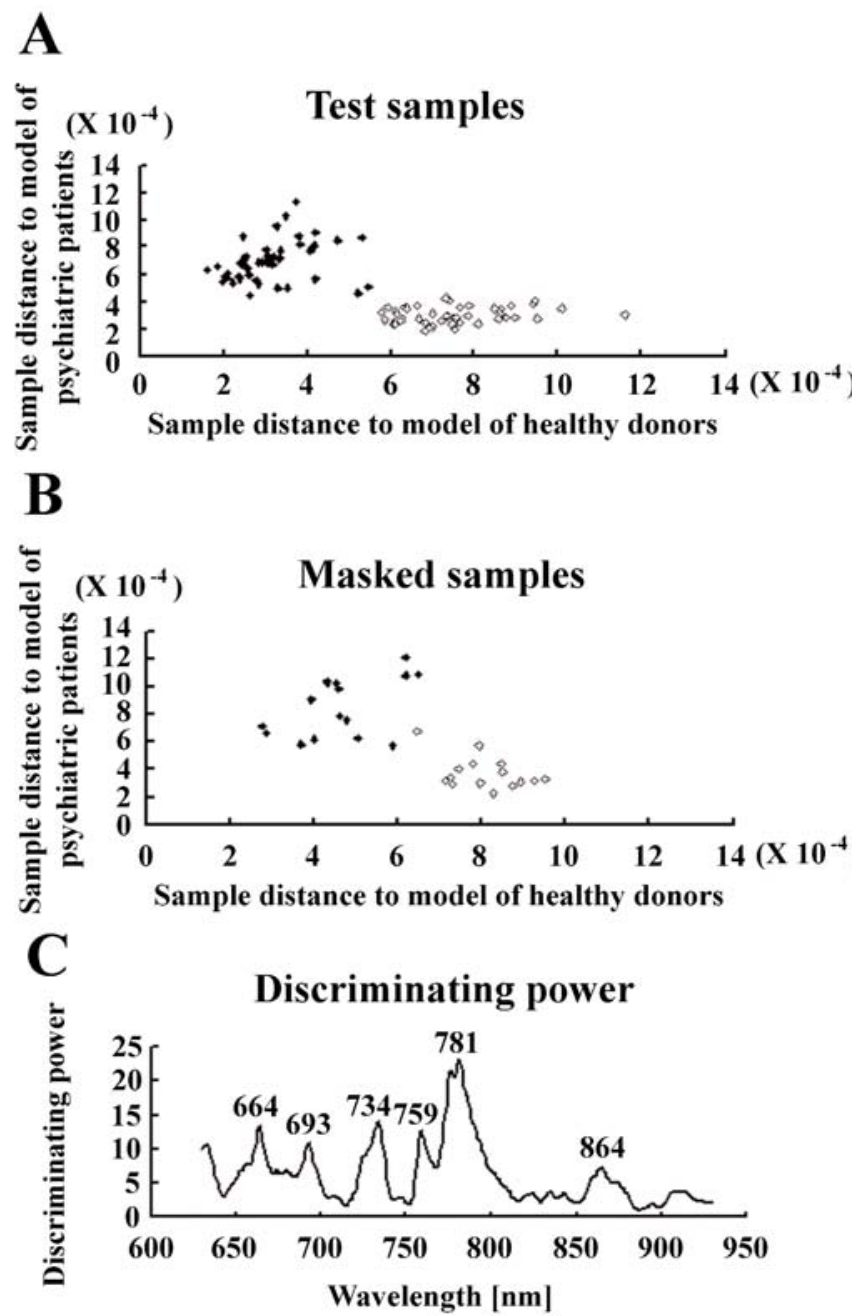

Figure 3. Soft modeling of class analogy (SIMCA) of the Vis-NIR calibration model for test samples and the predictions for masked samples using plasma of psychiatric and healthy donors. Vis-NIR spectral data of plasma samples from healthy donors and psychiatric patients were preprocessed by mean centering and smoothing and subjected to SIMCA calibration modeling to develop a multivariate model to manifest the characteristics of psychiatric patient plasma. A Coomans plot of SIMCA demonstrating that the healthy donor class (closed diamonds) and psychiatric patient class (open diamonds) of test samples (A) and masked samples (B) did not share multivariate space. (C) Discriminating power from the SIMCA calibration model shows several important peaks separating the plasma of psychiatric patients from that of healthy donors. unmedicated patient but could be clearly separated from healthy donors. Furthermore, the affected period and medication of psychiatric patients did not have any significant correlation with PC3 and PC4 scores or distances of each sample to the model of healthy donors in the Coomans plot (data not shown). These results indicate that drugs and affected periods did not influence the discrimination. On the other hand, it should be mentioned that complications with mental disorders, such as hypertension, diabetes mellitus, cancer, and so on, were not considered.

Using these reliable models, the spectral information modelled by PCA and SIMCA was extracted by the corresponding loadings and discriminating power, respectively. Concerning PC3, the loadings positively peaked at $\sim 730$ and $770 \mathrm{~nm}$, negatively peaked at $\sim 870 \mathrm{~nm}$ and $910 \mathrm{~nm}$, were slightly high at $\sim 690-810 \mathrm{~nm}$ and were slightly low at $\sim 810$ 


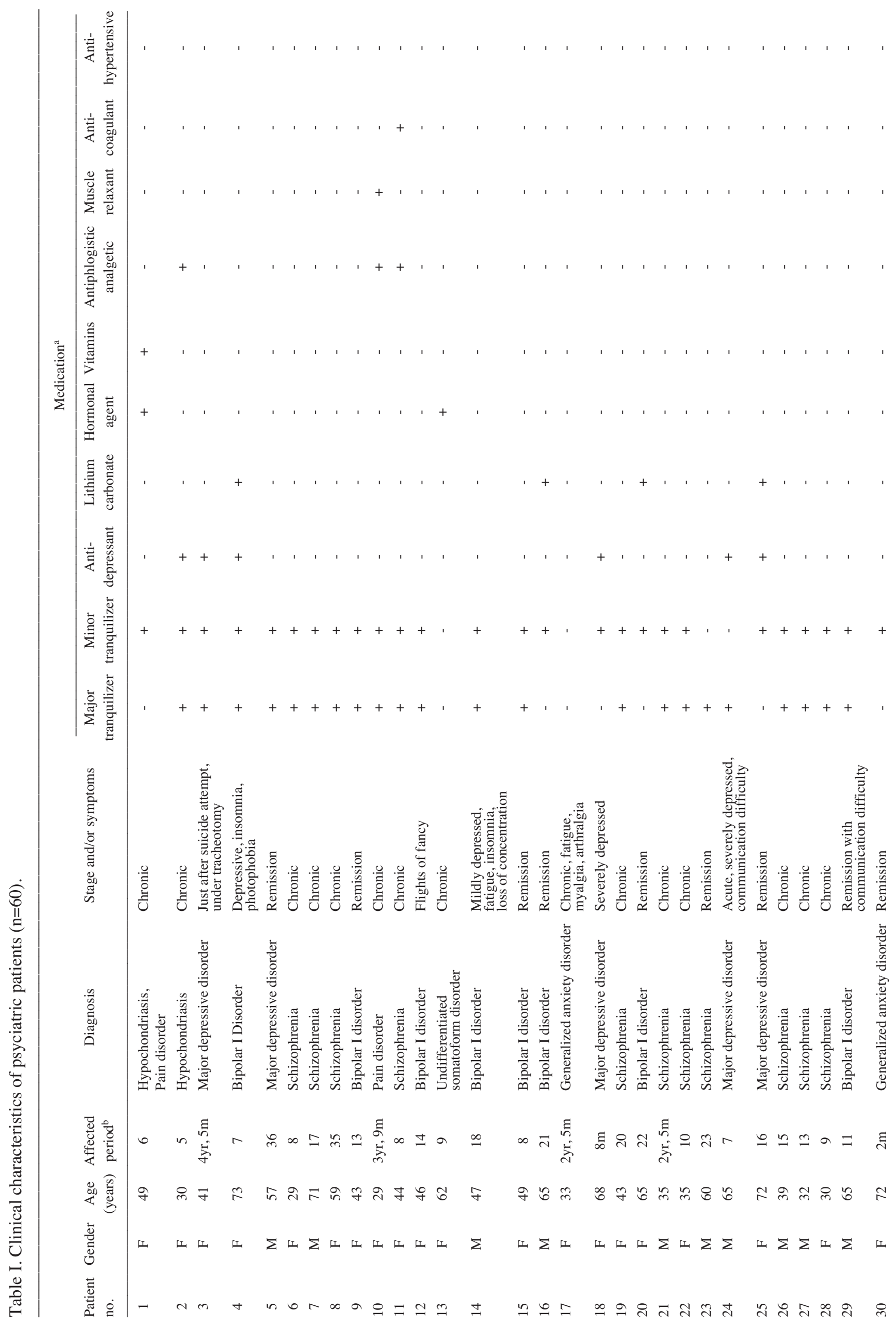




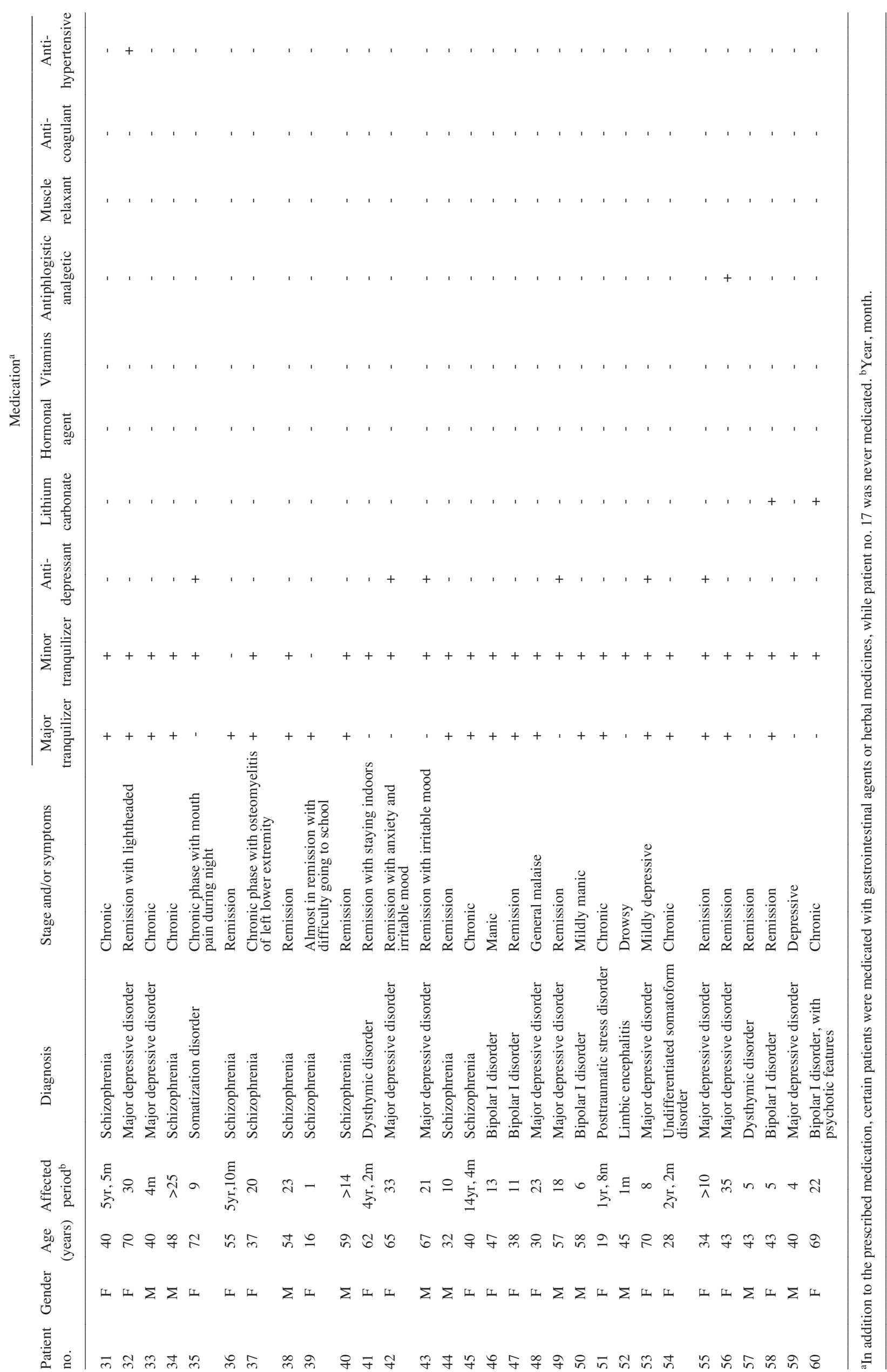


Table II. Possible assignment of peaks in discriminating power of the SIMCA model.

\begin{tabular}{lll}
\hline Wavelength $(\mathrm{nm})$ & \multicolumn{1}{c}{ Assignment } & References \\
\hline 664 & Unknown & - \\
693 & 4th overtone of superoxide tetrahydrate $\mathrm{O}_{2}-\left(\mathrm{H}_{2} \mathrm{O}\right) 2(696.8 \mathrm{~nm})$ \\
734 & OH stretch, 3rd overtone of $\mathrm{ROH}(738 \mathrm{~nm})$ \\
759 & $\mathrm{~N}-\mathrm{H}$ stretch, 3rd overtone of amides $($ secondary, bonded; one band $)(750-800 \mathrm{~nm})$ \\
& $\mathrm{O}-\mathrm{H}$ stretch, 3rd overtone of $\mathrm{H}_{2} \mathrm{O}(760 \mathrm{~nm})$ \\
781 & $\mathrm{~N}-\mathrm{H}$ stretch, 3rd overtone of $\mathrm{RNH}_{2}(779 \mathrm{~nm})$ \\
& $\mathrm{N}-\mathrm{H}$ stretch, 3rd overtone of amides (primary, ponded; two bonds) $(\sim 788 \mathrm{~nm})$ \\
& $\mathrm{C}-\mathrm{H}$ stretch, 3rd overtone of ArCH. (Aromatics) $(857-890 \mathrm{~nm})$
\end{tabular}

$890 \mathrm{~nm}$ (Fig. 2C). PC4 loadings positively peaked at $\sim 690$, 750,870 , and $910 \mathrm{~nm}$, negatively peaked at $\sim 770$ and $800 \mathrm{~nm}$, were slightly high at $\sim 650-720$ and $830-890 \mathrm{~nm}$, and were slightly low at $\sim 760-830 \mathrm{~nm}$ (Fig. 2C). The most prominent discriminating power, which represents independent variables (wavelengths) important in discriminating two classes (psychiatric and healthy), were peaks at $\sim 660,690,735,760$, 785 and $865 \mathrm{~nm}$ (Fig. 3C). The PCA loadings were generally consistent with the discriminating power of the SIMCA model, except for loadings of 950-nm peaks (Figs. 2C and 3C).

\section{Discussion}

Although the knowledge of psychiatric disorders has expanded and the need for research has become increasingly more important after Engel proposed his bio-psycho-social model in 1977 (1), progress in diagnostic procedures in the field of psychiatry has been slower than was hoped. The main reason for this is the biological, environmental and genetic complexity of psychiatric disorders, whose etiologies remain unknown. Recent study has shown the association between a specific allele of the $5^{\prime}$ promoter of the serotonin transporter gene and a high risk for developing depression in response to stress, but this represents only the tip of a gigantic iceberg in terms of our understanding of how biological, environmental and genetic factors interact to produce both healthy and psychiatric differences (12). Therefore, presently, a descriptive classification is used for diagnosis, which takes a long time period in which to reach a final decision, resulting in a delay in early treatment intervention.

Recently, the possibility of diagnosing viral infections (13), chronic fatigue syndrome (CFS) (14), and anti-phospholipid antibodies (15) by Vis-NIR spectroscopy combined with multivariate analysis has been reported. Vis-NIR radiation is absorbed due to the combination and overtone of vibrations such as the stretching and bending of hydrogenbearing functional groups including C-H, N-H and O-H (16), which are included in most biomolecules. Therefore, the spectral change in the Vis-NIR region reflects the change of biomolecules in samples.

The current descriptive method for the diagnosis of psychiatric disorders has not been performed objectively.
The findings of this study indicate the possibility of a new objective diagnostic approach using Vis-NIR spectroscopy, since a specific spectral pattern was found in plasma samples of psychiatric patients. The possible band assignment of discriminating power in the SIMCA model suggests that the spectral change is due to altered superoxide (17), amine (18), and primary and secondary amides (19) in plasma (Table II). Recent study has shown that dysfunction of the endocrine system in the hypothalamic-pituitary-adrenal axis is common in psychiatric patients (20). It would be of interest to analyze whether the spectral change observed in this study is related to neuroendocrine mechanisms. Further information obtained from the detailed analysis of Vis-NIR spectra may make significant contributions not only to the diagnosis but also to the understanding of the pathogenesis of psychiatric disorder.

In our previous study, we showed that sera of CFS patients could be distinguished from sera of healthy donors (14). Notably, the peaks distinguishing the CFS patients from healthy donors (14) were different from those discriminating psychiatric patients from healthy donors in the present study. These observations suggest that psychiatric disorders share some factors different from CFS and may provide some additional information on the controversial issue of whether psychiatric disorders are comorbidities of CFS $(21,22)$. Finally, it should be mentioned that instability and low between-run reproducibility of Vis-NIR spectra of liquid samples were sometimes noted. Temperature, water vapor, instrumental noise, and sampling errors would be considered the source of these problems. Therefore, great attention to minimize these factors should be paid during Vis-NIR measurements. Significant improvement to overcome these obstacles is necessary before the practical use of this method in diagnosis.

In conclusion, our findings suggest that plasma of psychiatric patients can be distinguished from that of healthy donors by Vis-NIR spectroscopy. The spectral change observed in psychiatric patients may provide a novel index of psychiatric disorders, suggesting Vis-NIR spectroscopy to be a promising tool with which to objectively diagnose psychiatric disorders. This may contribute to the early medical intervention of psychiatrics disorders; however, there remain obstacles such as the instability of Vis-NIR spectra which need to be addressed before its practical use. 


\section{Acknowledgements}

We thank Dr Hirohiko Kuratsune (Clinical Center for Fatigue Science, 21st Century COE Program, Osaka City University Graduate School of Medicine, Osaka, Japan) for discussions and the kind provision of plasma samples from healthy donors. This work was partly supported by Grants-in-Aid from the Japan Society for the Promotion of Science, Heiwa Nakajima Foundation, and Japan Science and Technology Agency.

\section{References}

1. Engel GL: The need for a new medical model: a challenge for biomedicine. Science 196: 129-136, 1977.

2. American Psychiatric Association: Diagnostic and Statistical Manual of Mental Disorders. 4th edition, American Psychiatric Association, Washington, DC, 2000.

3. World Health Organization: The ICD-10 Classification of Mental and Behavioural Disorders. Clinical Descriptions and Diagnostic guidelines. World Health Organization, Geneva, 1991.

4. Howsepian AA: The DSM-IV-TR 'Glossary of Technical Terms': a reappraisal. Psychopathology 41: 28-34, 2008.

5. Chiu E: Limitations in the current classification systems for dementia. Int Psychogeriatr 17 (Suppl 1): 17-26, 2005.

6. Strain JJ: Psychiatric diagnostic dilemmas in the medical setting. Aust NZ J Psychiatry 39: 764-771, 2005.

7. Savitzky A and Golay MJE: Smoothing and differentiation of data by simplified least squares procedures. Anal Chem 36: 1627-1639, 1964.

8. Barnes RJ, Dhanoa MS and Lister SJ: Standard normal variate transformation and de-trending of near-infrared diffuse reflectance spectra. Appl Spectrosc 43: 772-777, 1989.

9. Jolliffe IT: Principal Component Analysis. Springer, New York, 2002.

10. Wold S: Pattern recognition by means of disjoint principal components models. Pattern Recog 8: 127-139, 1976.

11. Coomans D, Broeckaert I, Derde MP, Tassin A, Massart DL and Wold S: Use of a microcomputer for the definition of multivariate confidence regions in medical diagnosis based on clinical laboratory profiles. Comput Biomed Res 17: 1-14, 1984.
12. Pezawas L, Meyer-Lindenberg A, Drabant EM, et al: 5-HTTLPR polymorphism impacts human cingulate-amygdala interactions: a genetic susceptibility mechanism for depression. Nat Neurosci 8: 828-834, 2005.

13. Sakudo A, Suganuma Y, Kobayashi T, Onodera T and Ikuta K: Near-infrared spectroscopy: promising diagnostic tool for viral infections. Biochem Biophys Res Commun 341: 279-284, 2006.

14. Sakudo A, Kuratsune H, Kobayashi T, Tajima S, Watanabe Y and Ikuta K: Spectroscopic diagnosis of chronic fatigue syndrome by visible and near-infrared spectroscopy in serum samples. Biochem Biophys Res Commun 345: 1513-1516, 2006.

15. Nojima J, Sakudo A, Hakariya Y, et al: Spectroscopic diagnosis of anti-phospholipid antibodies by visible and near-infrared spectroscopy in SLE patients' plasma samples. Biochem Biophys Res Commun 362: 522-524, 2007.

16. Murray I: Forage analysis by near infra-red spectroscopy. In: Sward Management Handbook. Davies A, Baker RD and Grant SA (eds). British Grassland Society, UK, pp 285-312, 1993.

17. Weber JM, Kelley JA, Nielsen SB, Ayotte P and Johnson MA: Isolating the spectroscopic signature of a hydration shell with the use of clusters: superoxide tetrahydrate. Science 287: 2461-2463, 2000.

18. Osborne BG, Fearn T and Hindle PT: Practical NIR Spectroscopy With Applications in Food and Beverage Analysis (Longman Food Technology). Longman Group United Kingdom, UK, 1993.

19. Workman J: Handbook of Organic Compounds: NIR, IR, Raman and UV-Vis Spectra Featuring Polymers and Surfactants. Academic Press, San Diego, CA, 2001.

20. Heinrichs M and Gaab J: Neuroendocrine mechanisms of stress and social interaction: implications for mental disorders. Curr Opin Psychiatry 20: 158-162, 2007.

21. Demitrack MA: Clinical methodology and its implications for the study of therapeutic interventions for chronic fatigue syndrome: a commentary. Pharmacogenomics 7: 521-528, 2006.

22. Ciccone DS and Natelson BH: Comorbid illness in women with chronic fatigue syndrome: a test of the single syndrome hypothesis. Psychosom Med 65: 268-275, 2003. 